\title{
Study on the Chilling Requirement and Dormant Physiology of Ziziphus jujuba 'Zhanshanmizao'
}

\author{
Qian Deng ${ }^{1, a}$, Yang Wang ${ }^{1, b}$, Wanqiu Zhu ${ }^{1, c}$, Yan Zhou ${ }^{1, d}$ \\ and Qunxian Deng ${ }^{1, \mathrm{e} *}$ \\ ${ }^{1}$ College of Horticulture, Sichuan Agricultural University, Chengdu, Sichuan, China \\ a635117027@qq.com, b1953457744@qq.com, czwq198995@qq.com, \\ dzhouyan6213500@qq.com, e1324856299@qq.com
}

${ }^{*}$ Corresponding author.

Keywords: Ziziphus jujuba 'Zhanshanmizao'; the chilling requirement model; the dormant physiology

Abstract. Seven-year-old tree of Ziziphus jujuba 'Zhanshanmizao' cultivated in field were used to study the degree dormancy, chilling requirement (CR) and dormant physiological indexes such as the content of water, carbohydrates, free amino acid, and soluble protein. The results showed that the $Z$. jujuba 'Zhanshanmizao' bud had a dormancy from early November, finished the natural dormancy from early January, then came into an enforce dormancy. Among $\leq 7.2^{\circ} \mathrm{C}$ model, $0-7.2^{\circ} \mathrm{C}$ model, and Utah model, $0-7.2^{\circ} \mathrm{C}$ model is suitable to be used for measuring the CR of Z. jujuba 'Zhanshanmizao' in Santai area, and the CR of Z. jujuba 'Zhanshanmizao' was $494 \mathrm{~h}$. The content of free water, soluble protein, and starch were after rising to decline, but bound water/free water ratio, the content of soluble sugar, and free amino acid were after the first drop and then up in the period of the natural dormancy.

\section{Introduction}

The important stage for the next cycle of growth and development of deciduous fruit trees is after meeting the requirement of low temperature and completing the natural dormancy [1]. The effective low temperature time required for deciduous fruit trees to break natural dormancy (internal dormancy) is called the cold demand of fruit trees and the low temperature demand [2]. Fruit dormancy is a complex physiological process. In recent years, more and more attention has been paid to the dormancy mechanism and biochemistry of buds and branches. So far, few people have studied the cold requirement and dormancy physiology of jujube trees. At the same time, there is no uniform and effective method to estimate the cold demand of deciduous fruit trees. Therefore, it is clear that Ziziphus jujuba 'Zhanshanmizao' is suitable for cooling and studying physiological and biochemical changes during dormancy period of Z. jujuba 'Zhanshanmizao'. In order to break or delay dormancy to promote or delay fruit ripening and to prolong the period of market, some measures, such as protected cultivation and dormancy agent, are used to break or delay dormancy. Increasing the income of fruit farmers has important guiding significance.

\section{Materials and Methods}

Materials. The tested material was 7 years old Z. jujuba 'Zhanshanmizao' (tillering propagation seedling), spaced at $2 \mathrm{~m} \times 3 \mathrm{~m}$. Orchard is located in Yongxin Zhen Yonglian Village, Santai County, Mianyang City. Orchard for hilly terraces, yellow red purple soil.

The end period of natural dormancy was determined by hydroponics [3]. From November 7 th, 2016 to February 30 th, 2017, 30 representative second branches of jujube were collected from the middle and upper part of the crown of jujube tree every 14 days. Take it back to the lab and put it in clean water. Then put it in an artificial climate box. Artificial climate box conditions: temperature $25{ }^{\circ} \mathrm{C}$, light cycle (day/night) $16 \mathrm{~h} / 8 \mathrm{~h}$, light intensity $3000 \mathrm{~lx}$, relative air humidity 50\% 70\%. Change water once in 2 days, at the same time cut the base of the branch $2 \mathrm{~mm}$. The germination rate after 20 days was regarded as the final germination rate. The germination percentage of jujube trees 
reached $70 \%$, which represented the natural dormancy of jujube trees was end. The sampling date was taken as the natural dormancy end period of Z. jujuba 'Zhanshanmizao'.

Methods. The temperature measurement is based on the result of automatic temperature recorder of meteorological bureau at Santai county, Mianyang city. According to the model of $\leq 7.2{ }^{\circ} \mathrm{C}$ [4], 0-7.2 ${ }^{\circ} \mathrm{C}$ and Utah [5], the low temperature demand of $Z$. jujuba 'Zhanshanmizao' was estimated. The conversion of the unit number of cold temperature to the number of hours of cold temperature in Utah model is shown in Table 1.The water content was determined by Li's method [6], the content of starch and soluble sugar was determined by anthrone colorimetry [6]. The free amino acid content was determined by ninhydrin colorimetry [7], and the content of soluble protein was determined by Coomassie brilliant blue dye binding method [7].

Statistical Analyses. Data analysis using Excel 2010.

Table 1 Transformation of temperature and chilling unit for Utah model

\begin{tabular}{cc}
\hline Temperature $\left({ }^{\circ} \mathrm{C}\right)$ & Chilling unit $(\mathrm{CU})$ \\
\hline 1.4 & 0 \\
$1.5-2.4$ & 0.5 \\
$2.5-9.1$ & 1.0 \\
$9.2-12.4$ & 0.5 \\
$12.5-15.9$ & 0 \\
$16.0-18.0$ & -0.5 \\
$>18.0$ & -1.0 \\
\hline
\end{tabular}

\section{Results and Discussion}

Germination rate. Between November 8 th, 2016 and February 28 th, 2017, the budding rate of $Z$. jujuba 'Zhanshanmizao' shoots was decrease at first, then risen (Table 2). Around December 20 th, 2016, the budding rate of Z. jujuba 'Zhanshanmizao' branches was the lowest, at $57.8 \%$, indicating that this was the deepest period of dormancy. Then the budding rate gradually increased, on January 3 th, 2017. The budding rate of Z. jujuba 'Zhanshanmizao' was 70.4\%, which indicated that the cold requirement of Z. jujuba 'Zhanshanmizao' was satisfied [8]. Thus, facility cultivation or use of sleep-breaking agent in early January is better.

Table 2 The budding rate of $Z$. jujuba 'Zhanshanmizao' under different chilling requirement

\begin{tabular}{cccccccccc}
\hline & \multicolumn{1}{c}{ Date (year - month - day) } \\
\cline { 2 - 10 } & $2016-$ & $2016-$ & $2016-$ & $2016-$ & $2017-$ & $2017-$ & $2017-$ & $2017-$ & $2017-$ \\
& $11-8$ & $11-22$ & $12-6$ & $12-20$ & $01-3$ & $01-17$ & $01-31$ & $02-14$ & $02-28$ \\
\hline Budding rate (\%) & 71.2 & 69.5 & 66.7 & 57.8 & 70.4 & 88.1 & 90.7 & 96.6 & 95.5
\end{tabular}

The accumulation of low temperature hour and low temperature unit. According to the model of $\leq 7.2{ }^{\circ} \mathrm{C}, 0-7.2^{\circ} \mathrm{C}$, and Utah, the accumulative low temperature of $502 \mathrm{~h}, 494 \mathrm{~h}$, and $499 \mathrm{CU}$ were obtained, respectively. The values of the three models are very close, and the values calculated by the model $\leq 7.2{ }^{\circ} \mathrm{C}$ are more similar to those calculated by the Utah model. The results show that $0{ }^{\circ} \mathrm{C}$ weather rarely occurs in autumn and winter in southern China, and the three models are applicable in Santai County. The result of this experiment is very close to that of 24 jujube varieties with a cooling requirement of $399 \mathrm{CU} 580 \mathrm{CU}$ by He [8].

moisture content. The free water content of jujube branch decreases first and then increases during natural dormancy (Fig.1). Free water was the lowest on December 13 th, 2016, at 10.6\%. The ratio of bound water to free water increased first and then decreased (Fig.2), reaching a maximum of 2.4 on December 13 th, 2016. The relationship between water and dormancy was studied [9], and it was suggested that reducing water supply, accumulation of carbohydrates and excess accumulation of carbohydrates could inhibit the effect of enzymes and lead to dormancy. In the deepest period of dormancy, the free water content and the total water content in the bud branch of jujube tree showed a 
downward trend, while the bound water showed an increasing trend, which indicated that the jujube tree could resist the bad environment in order to strengthen its ability to resist the bad environment. To avoid ice formation between cells, but to reduce its metabolic activity and water content, enhance cold resistance.

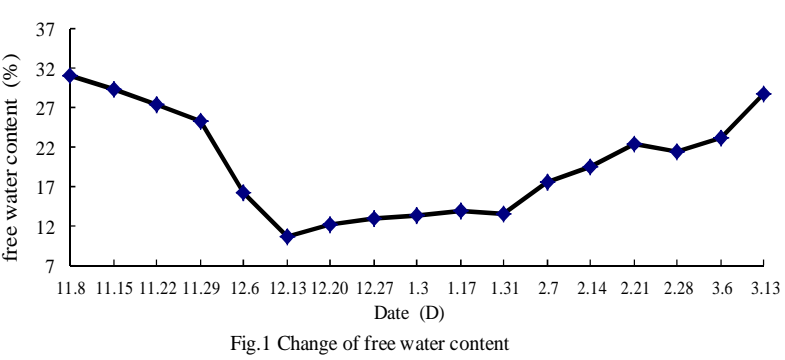

Fig. 1 Change of free water conten

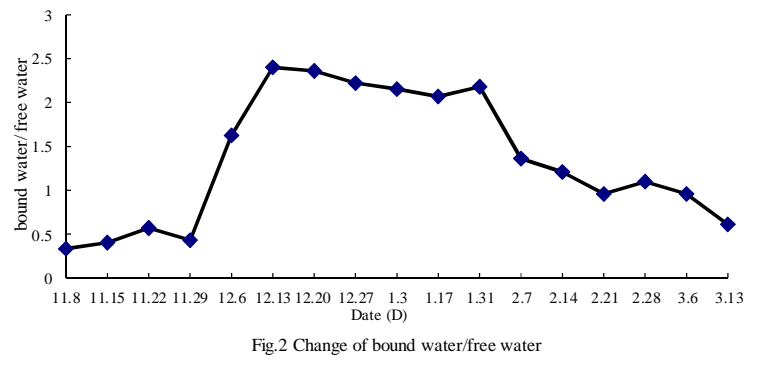

Fig. 2 Change of bound water/ free water

Carbohydrate content. The content of soluble sugar in the branches of $Z$. jujuba 'Zhasnhanmizao' increased first and then decreased during dormancy (Fig.3). The content of soluble sugar increased before December 13 th, 2016, and reached a maximum value of $4.74 \%$ on December 20 th. On February 14 th the following year, the minimum value was $1.62 \%$. The content of soluble sugar in the branch of Z. jujuba 'Zhanshanmizao' was on the rise after February 14 th of the following year. The change trend of starch content in Z. jujuba 'Zhanshanmizao' branch decreased first and then risen (Fig.4). On December 13 th, 2016, and February 14 th, 2016, the minimum and maximum values were 1.85 and 3.10, respectively. The change trend is consistent with the research result of Gao [9]. With the decrease of temperature, starch is converted to sugar, which is beneficial to increase the concentration of cell fluid and cell osmotic pressure, increased the concentration of protoplasm, thus reducing the freezing point and improve the cold resistance. Then there was a process of decrease, which was due to the gradual release of dormancy. The second rise was because the starch was converted into sugar, which could provide material and energy for physiological metabolism of jujube, and create conditions for bud germination. During dormancy, the starch content in the bud branch of jujube showed a reverse trend. During the winter, the amylase activity was activated with the decrease of temperature. The starch was transformed into soluble sugar, the freezing point was reduced, and the cold resistance was improved.

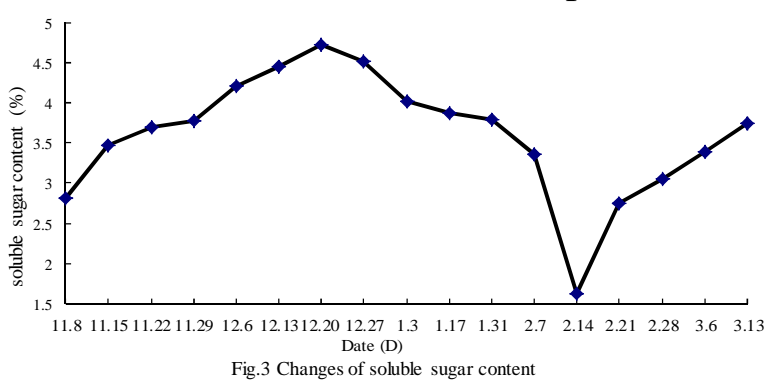

Fig.3 Change of soluble sugar content

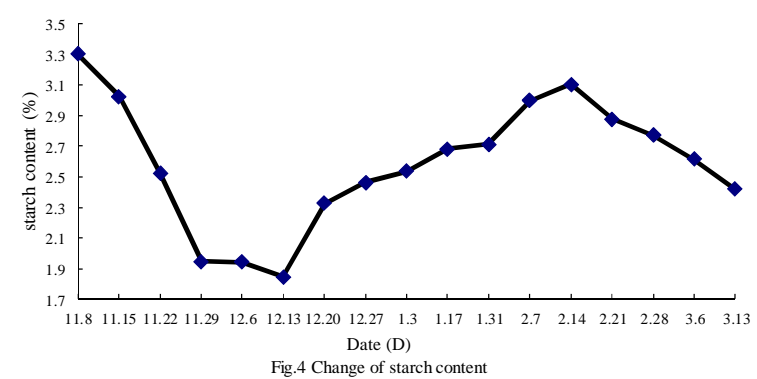

Fig.4 Change of starch content

Free amino acid content and soluble protein content. The trend of free amino acid content in branches of Z. jujuba 'Zhanshanmizao' was increased first and then decreased and then increased (Fig.5). On December 13 th, the content of free amino acids was the highest, with the content of $2326.34 \mathrm{~g} / \mathrm{g}$. The following year February 14 th is the lowest value, the lowest value is $503.20 \mathrm{~g} / \mathrm{g}$. The content of soluble protein in tree branch of Z. jujuba 'Zhanshanmizao' decreased first and then increased (Fig.6), the lowest value was $1.47 \mathrm{mg} / \mathrm{g}$ on December $13 \mathrm{th}$, and the maximum value was $3.23 \mathrm{mg} / \mathrm{g}$ on February $14 \mathrm{th}$. The change trend is consistent with the research result of Liu [10]. During natural dormancy, the content of free amino acids increased, the cell concentration and osmotic pressure increased, which led to the decrease of freezing point, and enhanced the cold resistance of Z. jujuba 'Zhasnhanmizao'. After forced dormancy, the soluble protein increased rapidly and the free amino acids decreased rapidly, which may be related to the rise of temperature. It provides a material basis for the new growth and development. 


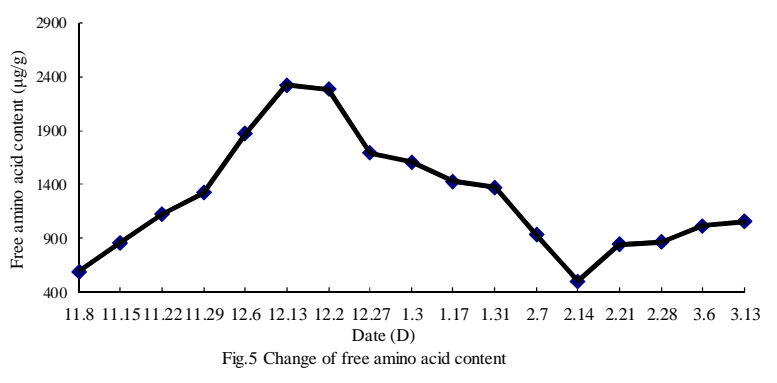

Fig.5 Change of free amino acid content

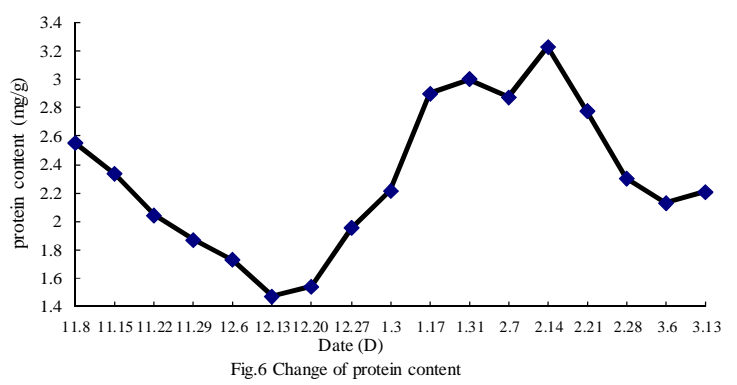

Fig.6 Change of protein content

\section{Conclusions}

The preliminary calculation shows that the chilling requirement of $Z$. jujuba 'Zhanshanmizao' is 494 $\mathrm{h}$, and the model is $0-7.2{ }^{\circ} \mathrm{C}$ model. The $Z$. jujuba 'Zhasnahnmizao' ended its natural dormancy in early January and entered into forced dormancy. With the deepening of dormancy, the free water content of jujube branch decreased and bound water/free water ratio increased during the natural dormancy period. During natural dormancy, the content of soluble sugar in the branches of $Z$. jujuba 'Zhansahnmizao' increased first and then decreased, while the starch content decreased first and then increased. During the period of forced dormancy, soluble sugar decreased and starch content increased rapidly. In the first ten days of January, the budding rate was over $70 \%$, and the soluble sugar content was close to the starch content. It could be used as a physiological index to judge the end of natural dormancy of Z. jujuba 'Zhanshanmizao', and provide the basis for application of dormancy breaking agent and shed.

\section{Acknowledgements}

This work was financially supported by the double subject construction plan of Sichuan Agricultural University and special action plan project of science, and technology rich people and strong county of Sichuan Province "Integration and demonstration of key technologies for industrialized development of Zizyphus jujuba 'Zhanshangmizao'of Santai County”.

\section{References}

[1] L.Z. Fu, H.L. Shen, L.R. Zou, L. Chen and J.H. Wen: Northern fruit Vol. 14 (2014), p. 1.

[2] W.B. Zhuang, Z. Zhang, T. Shi, P.P. Wang, J. Shao, X.Y. Luo and Z.H. Gao: Journal of Fruit Science Vol. 29 (2012), p. 447.

[3] H. Liang: South China Fruits Vol. 36 (2007), p. 74.

[4] J.H. Weinberger: Proc Amer Soc Hort Sci Vol. 56 (1950), p. 122.

[5] E.A. Richardson, S.D. Seeley and D.R. Walker: HortScience Vol. 9 (1974), p. 331.

[6] H.S. Li: The Principle and Technology of Plant Physiology and Biochemistry Experiment (Higher Education Press, Beijing 2000).

[7] Q.E. Xiong: An Experimental course in Plant Physiology (Sichuan Science and Technology Press, Chengdu 2003).

[8] R.P. He, J. J Du, F. Zhao, J. Li and R.S. Niu: Journal of Fruit Science Vol. 21 (2004), p. 182.

[9] C.Y. Gao, A. Zhang, Y.L. Fang, Z.W. Zhang and Q.H. Jin: Acta Botanica Boreali- Occidentalia Sinica Vol. 6 (2009), p. 1200.

[10]G.Q. Liu, S.T. He, J.P. Liu, R. Zhang and J.W. Zeng: Journal of Yunnan Agricultural University (Natural Science) Vol. 33 (2018), p. 90. 